\title{
Neoformación subescapular blanda con telangiectasias
}

\section{Soft subscapular neoformation with telangiectasias}

\author{
Juan Ramón José de Jesús Trejo-Acuña, ${ }^{*}$ José Alberto Ramos-Garibay, ${ }^{\ddagger}$ \\ Karla Patricia Estrada-Ramírez, $\$$ María Teresa Rosas-Morett $₫$
}

\section{CASO CLÍNICO}

Se trata de un hombre de 37 años, originario y residente de la Ciudad de México, quien acude a consulta por una dermatosis localizada a tronco, la cual afectaba la región subescapular izquierda, constituida por una neoformación subcutánea, de $1 \times 1.3 \mathrm{~cm}$ hemiesférica, pediculada, del color de la piel, de consistencia blanda, de bordes bien definidos, y con telangiectasias en su superficie, de evolución crónica, asintomática. (Figura 1). A la dermatoscopía sólo se observan áreas color rosa pálido y numerosos vasos lineales ramificados (Figura 2). Refería 10 meses de evolución. Sin tratamiento previo. Resto de piel y anexos sin alteraciones. Se realizó una biopsia escisional, la cual mostró una epidermis de características normales, dermis

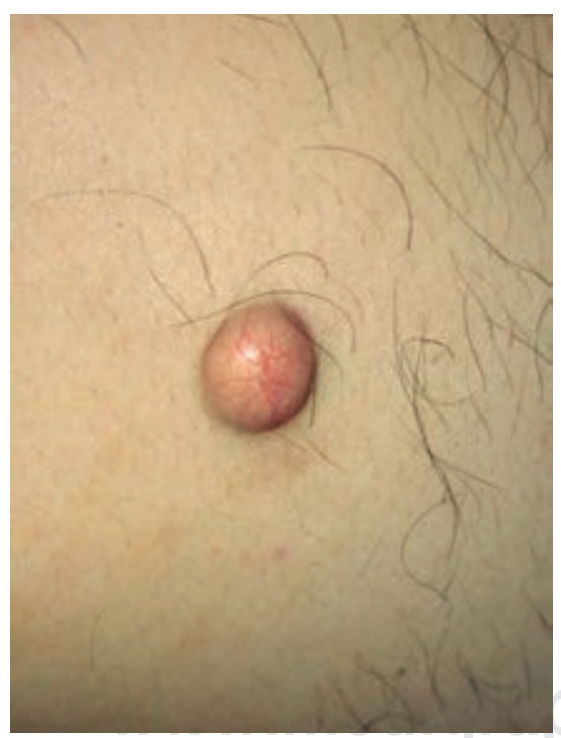

Figura 1:

Neoformación hemiesférica, del color de la piel, de consistencia blanda, pediculada, con numerosas telangiectasias en su superficie.
* Dermatólogo.

‡ Dermatopatólogo.

$\S$ Residente del tercer año de Dermatología.

" Residente del primer año de Dermatología.
Citar como: Trejo-Acuña JRJJ, Ramos-Garibay JA, Estrada-Ramírez KP, RosasMorett MT. Neoformación subescapular blanda con telangiectasias. Rev Cent Dermatol Pascua. 2020; 29 (2-3): 94-96. https://dx.doi.org/10.35366/99923

Centro Dermatológico "Dr. Ladislao de la Pascua», SSCDMX. 
reticular con fibras de colágena que se entremezclan con células adiposas. Resto del corte sin alteraciones (Figuras 3 a 5).

Con los datos antes proporcionados, ¿cuál es su diagnóstico?

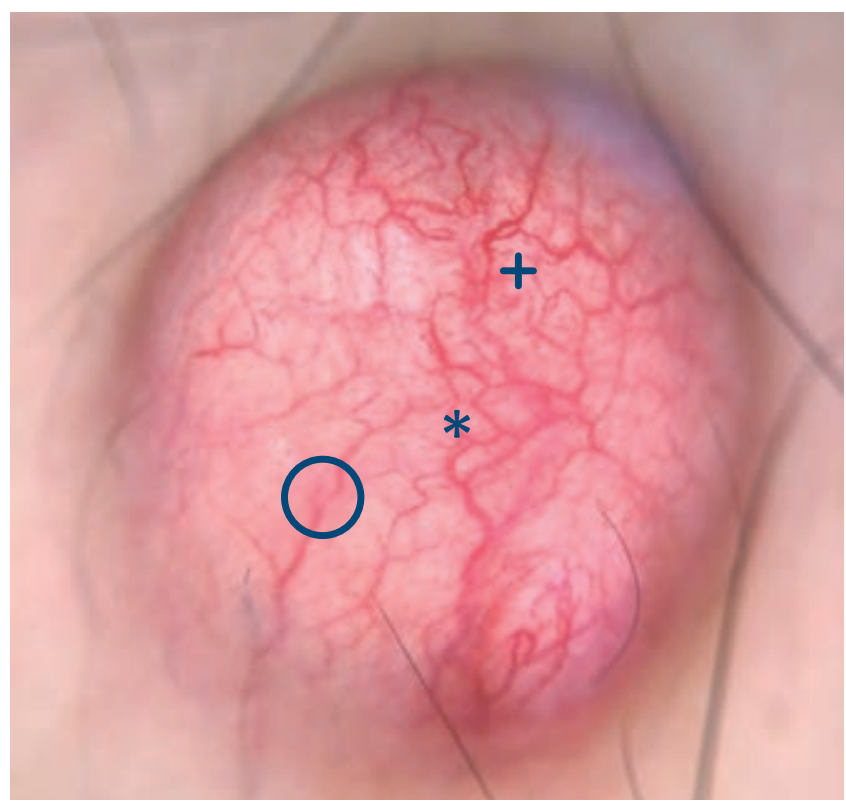

Figura 2: Imagen dermatoscópica. Numerosos vasos lineales irregulares ramificados $\left(^{*}\right)$, aislados en horquilla $(+)$ y áreas rosadas sin estructuras (círculo).

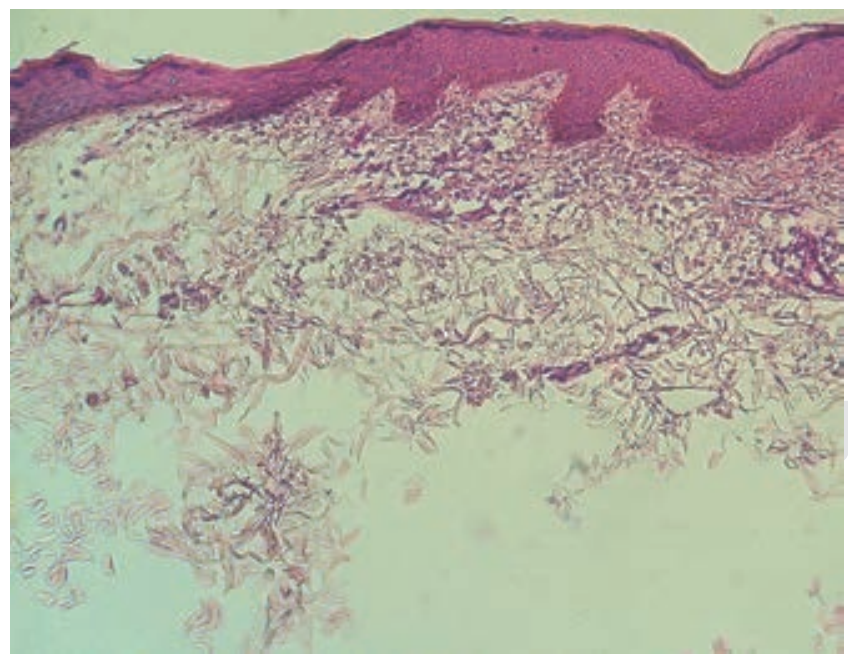

Figura 3: Epidermis sin alteraciones. Dermis reticular con fibras de colágeno. Imagen histológica (H\&E 4X).
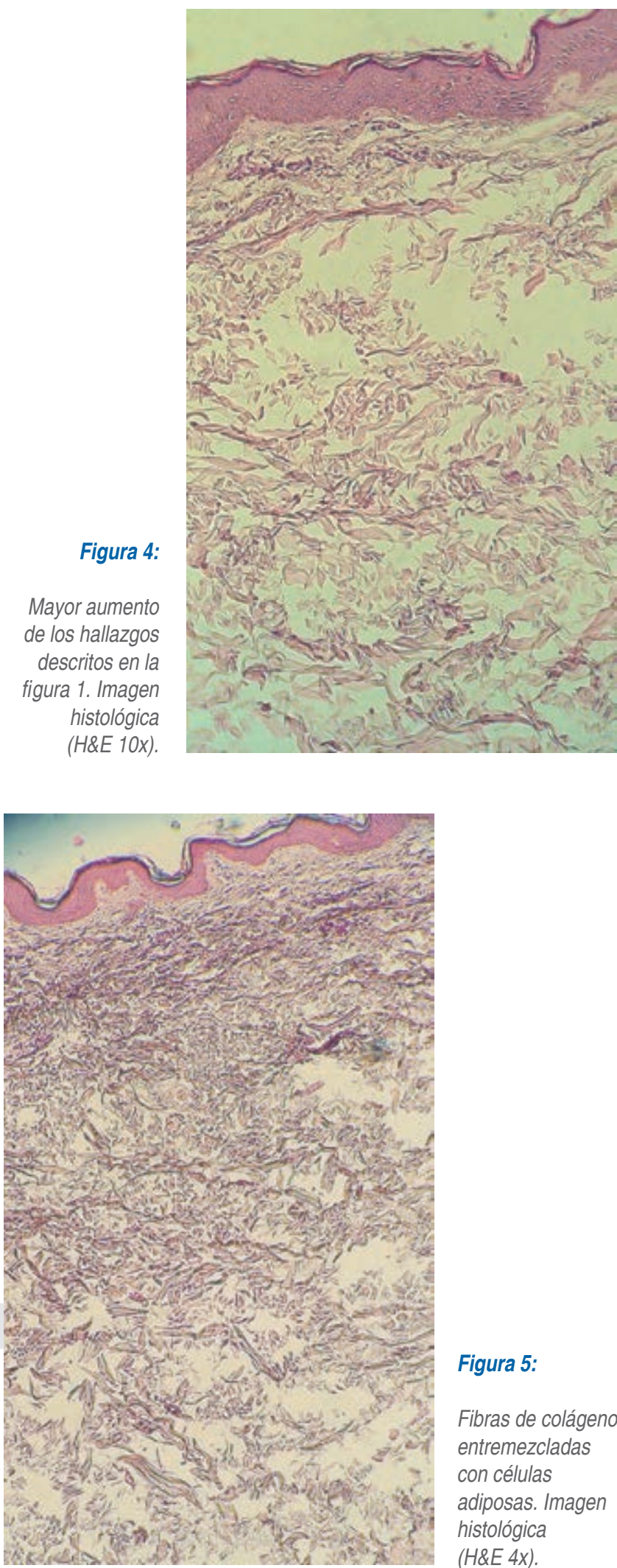

Figura 5:

Fibras de colágeno entremezcladas con células adiposas. Imagen histológica (H\&E 4X). 


\section{DIAGNÓSTICO: FIBROLIPOMA}

El fibrolipoma (FL) es una variedad histopatológica poco frecuente del lipoma. Los lipomas son tumores benignos constituidos por tejido adiposo maduro, agrupados en lóbulos divididos por septos, que se pueden presentar de forma superficial o profunda. ${ }^{1-3}$ Los FL se caracterizan a nivel histopatológico por estar constituidos por abundante tejido fibroso. ${ }^{2}$ Un estudio previo publicó que los FL constituyen $0.3 \%$ de los tumores benignos de la piel. ${ }^{4}$

Son más frecuentes en hombres adultos entre la quinta y sexta décadas de la vida y, por el contrario, poco frecuentes en menores de 20 años de edad. ${ }^{3}$

La etiología del FL es aún desconocida. Se han descrito varios mecanismos posibles, por ejemplo: acúmulo de nidos embrionarios de células lipoblásticas, metaplasia de células musculares, y degeneración grasa. ${ }^{5}$ Existen dos hipótesis: la congénita y la adquirida. La congénita lo atribuye a un factor genético causado por un desequilibrio endocrino que concluye con la formación de un tumor fibromatoso degenerado, o por la formación del mismo por lipoblastomatosis. ${ }^{2,4,6}$ La teoría adquirida involucra la diferenciación de células mesenquimales multipotenciales dando lugar a metaplasia en un lipoma preexistente, todo lo anterior secundario a un traumatismo local o isquemia prolongada. ${ }^{4,5}$ Es importante mencionar que metabólicamente las células grasas del FL son distintas de las células grasas normales, ya que las primeras no se utilizan para la producción de energía. ${ }^{5}$

Los hallazgos clínicos del FL varían dependiendo de su localización y tamaño. Generalmente se manifiesta como una neoformación subcutánea, del color de la piel o amarillenta, tamaño variable, de forma oval, con bordes bien definidos, superficie lisa, de consistencia blanda o firme, de crecimiento lento. Cuando compromete la cavidad oral, suelen observarse vasos sanguíneos en su superficie. ${ }^{2,6}$ Existen publicaciones de FL sésiles. ${ }^{7}$ El principal sitio de afectación es la mucosa oral, seguido de la lengua. ${ }^{6,7}$ La presentación suele ser asintomática, y los pacientes acuden a consulta por presentar una masa palpable. Rara vez refieren dolor o disfunción muscular. ${ }^{5}$

El diagnóstico definitivo se lleva a cabo mediante el estudio histopatológico, en el cual se aprecia una neoformación constituida por adipocitos maduros y bandas compactas de tejido conectivo, secundaria a un incremento progresivo del tejido fibroso. A diferencia del lipoma, se puede o no observar una cápsula con hialinización del tejido fibroso. ${ }^{1,8,9}$ Las bandas fibrosas pueden mostrar una pseudoinfiltración focalizada. ${ }^{7}$
El componente fibroso sólo se aprecia si el tumor es encapsulado. ${ }^{7}$

Aunque los FL son tumores benignos, existen reportes de conversión a liposarcoma. ${ }^{2,8}$

El tratamiento de elección es la extirpación completa de la lesión. Se ha observado que las lesiones fuera de cavidad oral presentan una tasa mayor de recidiva. . $^{6,10}$

El tratamiento se lleva a cabo principalmente con fines cosméticos, pues no ocasiona daño funcional. ${ }^{4,8}$

\section{COMENTARIO}

El FL es una variedad histopatológica poco frecuente de lipoma. El caso que se comunica en este artículo tenía una presentación clínica poco frecuente, con un crecimiento lento y progresivo. Se extirpó totalmente y hasta el momento no ha tenido recidiva. El tratamiento quirúrgico es la terapia de elección y la histopatología fue la clave para su diagnóstico.

\section{REFERENCIAS}

1. Lee SY, Jung SN, Sohn WI, Kwon H, Yoo G. Submuscular fibrolipoma of the forehead. J Craniofac Surg. 2010; 21: 1993-1994.

2. Corredor-Osorio R, Ramos-Pineda N, Orellana ME. Fibrolipoma on upper eyelid in child. GMS Ophthalmol Cases. 2016; 6: Doc03.

3. Stock N. Adipocytic tumors. Ann Pathol. 2015; 35: 41-53.

4. Shin SJ. Subcutaneous fibrolipoma on the back. J Craniofac Surg. 2013; 24: 1051-1053.

5. Kumaraswamy SV, Madan N, Keerthi R, Shakti S. Lipomas of oral cavity: case reports with review of literature. J Maxillofac Oral Surg. 2009; 8: 394-397.

6. Iwase M, Saida N, Tanaka Y. Fibrolipoma of the buccal mucosa: a case reportand review of the literature. Case Rep Pathol. 2016;2016:5060964.

7. laconetta G, Friscia M, Cecere A, Romano A, Dell'Aversana OG, Califano L. Rare fibrolipoma of the tongue: a case report. J Med Case Rep. 2015; 9: 177.

8. Jung SN, Shin JW, Kwon H, Yim YM. Fibrolipoma of the tip of the nose. J Craniofac Surg. 2009; 20: 555-556.

9. Patterson JW. Weedon's skin pathology. 4th ed. (Livingstone/Elsevier C, ed.). Edinburgh Churchill Livingstone Elsevier, 2016. doi: 10.1097/ pap.0b013e3182026f09.

10. Fregnani ER, Pires FR, Falzoni R, Lopes MA, Vargas PA. Lipomas of the oral cavity: clinical findings, histological classification and proliferative activity of 46 cases. Int J Oral Maxillofac Surg. 2003: 32: 49-53.

\author{
Correspondencia: \\ Dr. Juan Ramón Trejo Acuña, \\ Dr. Vértiz Núm. 464 Esq. Eje 3 Sur, \\ Col. Buenos Aires, 06780, \\ Alcaldía Cuauhtémoc, CDMX. \\ Tel. 5519-6351. \\ E-mail: hermesjuanderma@hotmail.com
}

